\title{
Application of three-dimensional printing for colon targeted drug delivery systems
}

\author{
Nitin B. Charbe, Paul A. McCarron'ㄹ, Majella E. Lane², Murtaza M. Tambuwala ${ }^{1}$ \\ Unit of Clinical Pharmacology, Luigi Sacco University Hospital, University of Milan, Milan, Italy, ${ }^{1}$ School of Pharmacy and Pharmaceutical \\ Sciences, Saad Centre for Pharmacy and Diabetes, Ulster University, Coleraine, Co. Londonderry, ${ }^{2}$ UCL School of Pharmacy, London, \\ England, United Kingdom
}

\section{Abstract}

Orally administered solid dosage forms currently dominate over all other dosage forms and routes of administrations. However, human gastrointestinal tract (GIT) poses a number of obstacles to delivery of the drugs to the site of interest and absorption in the GIT. Pharmaceutical scientists worldwide have been interested in colon drug delivery for several decades, not only for the delivery of the drugs for the treatment of colonic diseases such as ulcerative colitis and colon cancer but also for delivery of therapeutic proteins and peptides for systemic absorption. Despite extensive research in the area of colon targeted drug delivery, we have not been able to come up with an effective way of delivering drugs to the colon. The current tablets designed for colon drug release depend on either pH-dependent or time-delayed release formulations. During ulcerative colitis the gastric transit time and colon pH-levels is constantly changing depending on whether the patient is having a relapse or under remission. Hence, the current drug delivery system to the colon is based on one-size-fits-all. Fails to effectively deliver the drugs locally to the colon for colonic diseases and delivery of therapeutic proteins and peptides for systemic absorption from the colon. Hence, to overcome the current issues associated with colon drug delivery, we need to provide the patients with personalized tablets which are specifically designed to match the individual's gastric transit time depending on the disease state. Three-dimensional (3D) printing (3DP) technology is getting cheaper by the day and bespoke manufacturing of 3D-printed tablets could provide the solutions in the form of personalized colon drug delivery system. This review provides a bird's eye view of applications and current advances in pharmaceutical 3DP with emphasis on the development of colon targeted drug delivery systems.

Keywords: Colitis, colon drug delivery, personalized medicine, three-dimensional printing

Address for correspondence: Dr. Murtaza M. Tambuwala, Saad Centre for Pharmacy and Diabetes, School of Pharmacy and Pharmaceutical Sciences, Ulster University, Coleraine, BT52 1SA, Northern Ireland, United Kingdom.

E-mail: m.tambuwala@ulster.ac.uk

\section{INTRODUCTION}

In August 2015, the US-Food and Drug Administration (FDA) gave approval for the first three-dimensional (3D)-printed pill Spritam. Spritam (levetiracetam), a drug, widely used

\begin{tabular}{|l|l|}
\hline \multicolumn{2}{|c|}{ Access this article online } \\
\hline Quick Response Code: & Website: \\
\hline & www.jpionline.org \\
\cline { 2 - 3 } & \\
\hline
\end{tabular}

to control seizures made by Aprecia Pharmaceuticals of Blue Ash, Ohio, using 3D-printed technology. ${ }^{[1]}$ Spritam now paved the way to utilize 3D printing (3DP) technology for the development of oral dosage form and drug delivery systems for human use. The company has developed

This is an open access article distributed under the terms of the Creative Commons Attribution-NonCommercial-ShareAlike 3.0 License, which allows others to remix, tweak, and build upon the work non-commercially, as long as the author is credited and the new creations are licensed under the identical terms.

For reprints contact: reprints@medknow.com

How to cite this article: Charbe NB, McCarron PA, Lane ME, Tambuwala MM. Application of three-dimensional printing for colon targeted drug delivery systems. Int J Pharma Investig 2017;7:47-59. 
Charbe, et al:: 3D printed colon drug delivery systems

an in-house technology based on ZipDose for the manufacture of Spritam. ZipDose was originally developed using a 3D platform that originated at the Massachusetts Institute of Technology. Aprecia achieved this landmark by fusing technology platform that combines pharmaceutical formulation science with 3DP. This fusion allowed the manufacturer to develop a highly porous tablet that disintegrates rapidly with a sip of liquid. This technology also helped the manufacturer develop the high-dose tablets; particularly important for the people who have difficulty in taking traditional tablets. 3D-printed constructs open up the whole new avenue of tailored dosage formulation based on individual patients' needs. Spritam success is based on the strategic application of the 3DP to the field of pharmaceutical sciences.

Currently, 3D printers are being used to create a wide variety of objects such as car parts, organs for transplants, fashion accessories, pharmaceuticals, and medical devices. In case of solid organ transplantation, 3D-printed organs could use patients own cells to create the new organ. Organ created by stem cells using 3DP technology could serve two important purposes: (1) no or negligible rejection chances and (2) no longer waiting list. $3 \mathrm{D}$ printers have the capacity to print medical devices with complex design, geometry, and features that match with individual patient's anatomy. The regulatory authorities has already authorized various 3D-printed medical devices. ${ }^{[2]}$ Previously available standard designs are used to create the multiple identical replicas of the same device. Personalized devices are created as per the patient's requirement confirmed by imaging data, for example, computerized tomography scan, magnetic resonance imaging scan. Medical devices created to take care of the situational and personal needs are called as personalized patient-matched or patient-specific devices. Currently, available 3D-printed healthcare devices include instruments, implants, and external prostheses.

In the field of pharmaceutical dosage formulations, 3DP not only holds the promise for dispensing on demand medication but also is useful in combining complex release profiles and geometries. The high degree of flexibility and control with 3DP not only enables the preparation of complex tailored dosage forms with different release profiles but also adds the precision parameter in personalized therapy. In future, this technology also offers unique opportunity for the manufacturing of the site-specific, sustained, or immediate drug delivery systems such as colon targeted drug delivery system personalized according to the patient's gastric transit time.

\section{TARGETING DRUG RELEASE IN THE COLON}

To avoid the nonspecific side effects due of the high drug concentration in stomach and small intestine, there is an increased interest in the development of the colon drug delivery system (CDDS) for disease localized to the colon. Drug delivery to the pathological site not only improves the therapeutic output but also helps avoid the side effect at the nonspecific site. Gastrointestinal tract (GIT)-specific drug delivery approach found to be very useful for the treatment of diseases such as ulcerative colitis, gastroenteritis, gastric ulceration, infectious diarrhea, gastrointestinal (GI) stromal tumors, Crohn's disease, amebiasis, colonic cancer, and local treatment of colonic ulceration. Delivering drugs to colon has several other advantages, such as colonic delivery is believed to be very useful for the systemic delivery of gene products such as protein, polypeptide drugs, and antibodies. The small intestine is considered as the primary drug absorption site, but gene products are generally poorly absorbed form it. Gene products are generally degraded by various enzymatic degradation process and lower $\mathrm{pH}$ of the small intestine and stomach, respectively. Proteolytic activity of colonic mucosa is not only mild but also less diverse when compared with the intestine. Colon $\mathrm{pH}$ condition is not adverse as that of the stomach. CDDS not only transports the drugs to the colon but also is an important tool to protect the gene products or acid liable drugs from the adverse condition. Despite the lower epithelial surface area of the colon compared with the small intestine, there are a number of drugs, such as simvastatin that are better absorbed in the colon due to the reduced enzyme activity and reduced P-glycoprotein efflux. ${ }^{[3]}$ All these factors stimulated the interest in the targeted delivery of the gene products and small molecules at the colon surface.

The cause of concern for the conventional tablets and powder-based capsules for colonic delivery is the low fluid level in the colon, usually not sufficient for disintegration and dissolution process. Normally, the level of fluid available for disintegration and dissolution is limited, with some reports of free water levels to be no more than a few milliliters being available. ${ }^{[4]}$ In this situation, it is advantageous if we could manage to deliver the drug in the form of liquid, for example, liquid-filled hard capsules, to protect the drugs from the harsh acidic condition of the stomach or from the proteolytic activity of the enzymes present in the small intestine.

To work efficiently, the CDDS should protect the drug core from the acidic environment of the upper GIT. In nutshell, neither drug absorption should occur in the 
stomach/small intestine, nor the bioactive agent should undergo degradation process at any of these sites. Drugs from CDDS are only expected to be absorbed once it reaches the colon. As discussed earlier, the colon is an appropriate absorption site for gene products such as peptides and protein primarily because (i) digestive enzymes are less active and less abundant and (ii) proteolytic activity of the enzymes present in the colon are less potent when compared with enzymes present in the small intestine. Thus, CDDS is very useful to protects peptide drugs from degradation, thereby releasing the drug into colon..$^{[5]}$ Finally, the colon is considered to be more sensitive to the absorption enhancers because of the higher residence time of about 5 days, thereby improving the chances of absorption of difficult to absorb drugs. ${ }^{[5]}$

Alternatively, colon delivery of the drugs is achievable through rectal route. Even though the rectal route avoids the first pass metabolism and is the quickest route for targeting the colon, colon targeted tablets through oral route are considered as the preferred method for colon delivery. The intra-rectal route is not only useful for delivery of drugs for systemic absorption, but can be used for the topical delivery of anti-inflammatory agents and corticosteroids for the treatment of ulcerative colitis. ${ }^{[6]}$ Although drugs such as prednisolone are absorbed from the large intestine, they are believed to be more effective if applied topically. ${ }^{[7]}$ For example, the unnecessary high concentration of prednisolone in the stomach and intestine increases the systemic absorption and leads to the side effects such as insomnia, headache, dizziness, and weight gain. Hence, targeting prednisolone to the colon surface in the conditions such as ulcerative colitis or Crohn's disease should be the preferred choice. Similarly, administration of dexamethasone and methylprednisolone by oral and intravenous routes produce severe side effects such as adenosuppression and immunosuppression. Targeted delivery to colon not only lowers the required dose of such drugs (e.g., dexamethasone and methylprednisolone) but also avoids the unwanted effects caused by high doses at the nonspecific site. ${ }^{[8]}$ Similarly, common side effect when mesalazine administered orally includes constipation, dyspepsia, abdominal cramps, nausea, and abdominal pain. However, these side effects are avoidable if mesalazine is delivered to the proximal colon in patients with inflammatory bowel disease. ${ }^{[0]}$

The amount of drug reaching the colon via the rectal route depends on formulation factors, flow of colonic contents and retention time. It is already demonstrated that enema has the superior capability to move against the direction of flow when compared with foam and suppositories.
This essentially proves that enema has the better-spreading capacity if compared with foam and suppositories. One of the problems associated with the colon absorption is relatively thick contents which not only hinders the mixing but also reduces the availability of the drug to the mucosal membrane.

The human gut is the home for around 100 trillion microorganisms of around 4000 distinct species which release several different types of enzymatic proteins. Gut microflora can vary from person to person and believes to depend on the bacterial flora of mothers. Gut microflora not only found to be directly related to diseases such as allergies and asthma but also has the role in variable drug absorption. Variable microflora could lead to variable intensity of the bacterial enzymes which ultimately could lead to the variation in the degradation of the enzymatic cleavages. Among the reactions carried out by this gut flora, the most important one includes azoreduction and enzymatic cleavage. ${ }^{[10]}$ These metabolic/degradation processes are vital for the metabolism of many drugs and bond cleavages. Knowledge of the specificity of the enzyme at the particular location of the gut is useful to develop the colon targeted delivery of acid or enzyme liable products, for example, gene products.

\section{pH AND MICROFLORA OF COLON}

During fasting state, the stomach $\mathrm{pH}$ usually remains constant between 1 and 2; however, it increases after a meal. The $\mathrm{pH}$ of around 6.5 and 7.5 is observed in the upper and lower small intestine, respectively. A significant drop in the acidity from ileum to colon has been noted. $\mathrm{pH}$ value of 5.7 and 6.4 has been noted in the ascending colon and cecum of a healthy individual, respectively. The $\mathrm{pH}$ of 6.6 and 7.0 is observed in the transverse and descending colon, respectively. This $\mathrm{pH}$ gradient across the GIT offers the unique opportunity for the development of the targeted drug delivery system. For example, polymer soluble at the $\mathrm{pH}$ of 6.6 and 7.0 is valuable to develop the CDDS. The $\mathrm{pH}$-dependent polymers for CDDS must be practically insoluble at lower $\mathrm{pH}$, but solubility should increase with the increase in $\mathrm{pH}$ value. ${ }^{[1]}$ The main function of the $\mathrm{pH}$-dependent polymer used for the CDDS is the protection of drug core in the stomach and intestine. In certain situation, some polymers may start to dissolve in the lower small intestine, thereby affecting the specificity of the formulation. In the case of the enteric-coated formulation, the decline in $\mathrm{pH}$ from the distal end of the small intestine to the colon could also result in problems such as lengthy lag times at the ileocecal junction or rapid transit through the ascending colon, ultimately affecting the site specificity of drug delivery. ${ }^{[11]}$ 
The microflora of the colon is in the range of 1011-1012 colony-forming unit/mL, consisting mainly of anaerobic bacteria, and belongs to the genus like genus Bacteroides, Gram-positive cocci. ${ }^{[12]}$ These bacteria not only derived their food/energy from the undigested materials such as polysaccharides, but some of them are found to be involved in the synthesis of useful vitamins. ${ }^{[13,14]}$ For the degradation of undigested materials, the microflora produces a vast number of enzymes such as glucuronidase (hydrolysis of $\beta$-D-glucuronic acid residues), xylosidase (hydrolysis of (1->4)-beta-D-xylans), arabinosidase (catalyse beta-L-arabinoside to L-arabinose), galactosidase (breaking of a glycosidic bond), nitroreductase (catalyze the reduction of nitro groups), and deaminase (catalyze the breakdown of nucleoside). ${ }^{[15]}$ These enzymes as mentioned earlier not only degrade the undigested polysaccharides but could also be used to develop the colon-specific drug delivery system. To develop such system, a substrate for the degradation by colonic enzymes would be the more precise approach. Such polymers not only shield the drug core from the acidic condition of the stomach and small intestine but also would release the drug after degradation by the enzymes present specifically in the colon. After reaching the colon, break down of the polymer backbone or its degradation could lead to the loss of its mechanical strength, thereby making them unable to hold the drug core. ${ }^{[16]}$ Colon-specific enzymes and conditions have already been exploited for the development of the various colon-specific drug formulations, for example, colon-specific prodrug, azo-polymeric prodrugs, polysaccharide-based drug delivery system. ${ }^{[17]}$

On the other side, it believed that a key factor for future improvements in disease treatment is the delivery of health care at the personalized levels, which already lead to the development of the concept of the personalized medicine/therapy. Personalized medicine is the tailored made therapy adjusted according to the patient needs and mostly based on genetic testing including proteomics and metabolomics analysis. Personalized therapy also requires proper medicine at unique dose through an appropriate route of drug administration. These personalized medicines hence need to be manufactured on the routine basis like its counterpart.

The most popular dosage form is the tablets taken orally. Tablet popularity is essential because of their universally adapted manufacturing process, good compliance, ease of administration, and fixed, accurate dosing. Powders can be compressed into tablets directly or after granulation (wet or dry). Tablet manufacturing involves several intermediate steps which include milling, mixing, and granulation (dry and wet). Any of these steps could be the problem source in tablet manufacturing, ultimately leading to the whole batch failure. Since the beginning of the modern medicine, almost all over the world, compressed tablets are manufactured at mechanized central plants. The process involved in the manufacturing of the tablets is clearly not suitable to the concept of personalized medicine/therapy. ${ }^{[18]}$ This is because the conventional method (wet and dry granulation and direct compress) provides the strict restriction on the variations in the dosage form (e.g., zero or linear release profiles and different shapes and sizes).

Liquid formulation is flexible in dose adjustment; however, personalization of the solid dosage forms, for example, tablets manufactured by the classical method is quite difficult. Personalized dosing with two-dimensional (2D) printing involves the direct lithography of drug-based ink on smooth flat biocompatible surfaces has been reported. ${ }^{[19]}$ This method involves the printing of several thousand dots of drugs and adjunct in the form of inks. Felodipine, a poorly soluble drug, was first printed using ink-jet printing technique. Polyvinylpyrrolidone has used an excipient in this method. From such formulations, the drug release could be modulated by increasing or decreasing the drug loading or by adding the adjuncts which could increase or decrease the release. As 2D printing is based on the microdeposits, a larger dose is not achievable and hence this approach is only confined to the most active drugs. In spite of some disadvantage, the $2 \mathrm{D}$ approach has explained the potential of $3 \mathrm{D}$ printers in a practical 3DP of dosage forms.

\section{THREE-DIMIENSIONAL-PRINTED TABLETS}

Precise drug delivery at the specific site is the key to the efficiency of the targeted drug delivery, for example, dissolution and disintegration in the colon are the keys to the success of the colon targeted tablet. Controlled release of oral dosage forms, specifically of tablets, is often acquired with the help of polymer coatings, semi-permeable membranes, and capsule. Drug release from the tablet across the different $\mathrm{pH}$ range of the GIT is achievable using different polymers soluble at different $\mathrm{pH}$. Same is achievable using specific chemical bonds cleavable by specific bacterial enzymes present across the GIT. The thickness of the polymer coat can be utilized to control the rate of water penetration into and drug diffusion out of the tablet.

Tablet fabrication techniques currently available are effective at controlling the release of a single drug or drug combination but are ineffective in controlling the release of 
multiple drugs independently across the various GI sites. In case of multiple drug administration, one must administer the multiple dosages independently. The solution to such problem is to manufacture the single tablet with multiple drugs. However, this is not the perfect solution in case if one drug needs to get release in stomach or intestine and other in the colon. The main hindrance in the formulation of such tablet is the complexity involved in accommodating the multiple drugs with multiple polymers in a single formulation. This problem of complexity is now overcome with the help of new sophisticated technique such as 3D printers. For example, 3D-printed tablet Spritam is already approved by the US-FDA and several other formulations are under investigation.

Khaled et al. used 3D extrusion printing technique to prepare five-in-one dose combination polypill with well-defined immediate release (IR) and sustained release (SR) profiles. ${ }^{[2]}$ This five-in-one dose polypill has combined pravastatin, atenolol, ramipril, aspirin, and hydrochlorothiazide in one single pill. Among them, aspirin and hydrochlorothiazide layers were present on the top of the $12 \mathrm{~mm}$ tablet for the IR, whereas the pravastatin, atenolol, and ramipril were separated in three SR compartments each separated by a hydrophobic cellulose acetate shell [Figure 1]. Cellulose acetate was used to act as a permeable carrier. Khaled et al. successfully utilized 3D extrusion printing to developed complex geometry as shown in Figures 1 and 2 . Authors have successfully proved that the $3 \mathrm{D}$ printer could be successfully used to prepare polypill to deliver five distinct drugs through two different and well-defined release mechanisms: IR and SR. Each drug in this polypill was successfully separated with the help of polymer cellulose acetate, thereby avoiding the incompatibility and allowing the maximum flexibility in handling the unique setting for each drug.

Sometimes, patients have to take more than two drugs at a time, for example, patient having diabetes and high

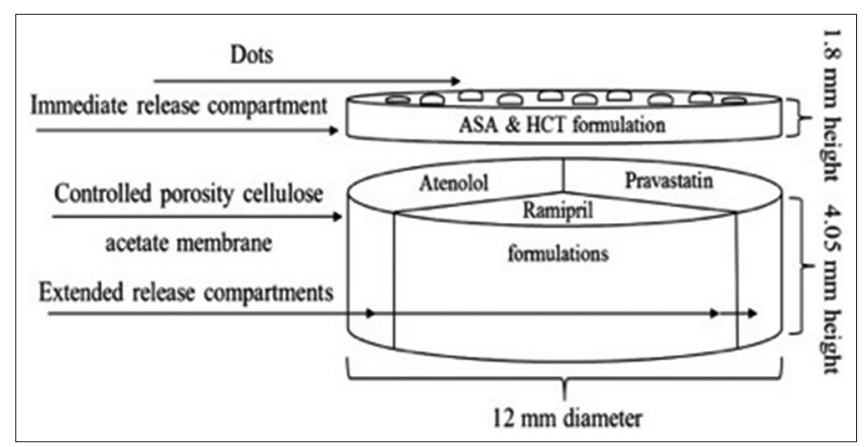

Figure 1: Structural diagram of the five-in-one polypill, where aspirin and hydrochlorothiazide are in the immediate release compartment and atenolol, pravastatin, and ramipril are in sustained release compartments. ${ }^{[20]}$ Reprinted with permission blood pressure, HIV infection. In such cases, increased adherence to the dose regimen is the key for the treatments. Formulations such as polypill not only add the convenience of a single tablet but also could help personalize the dosages and release for each drug independently. This polypill could be of enormous importance in prevention and treatment of heart-related disorders, HIV, etc.

Most recently, prefabricated polyvinyl alcohol (PVA) filaments have been utilized as drug carriers for the fabrication of tablet by fused deposition modeling (FDM) printers. ${ }^{[1-23]} \mathrm{FDM}$ is the new exciting low-cost solution for fabricating personalized formulations with controlled release profiles. Goyanes et al. were the first to evaluate the use of FDM with the incorporation of a model drug (fluorescein) into commercially available PVA filaments and the printing of tablets of different infill percentages. ${ }^{[2]}$ PVA filaments were swelled in the solution (ethanol) of the drugs for a different time to get the different loading. No chemical interaction between drugs and the filament and no change in the diameter of filament before and after the swelling were observed. Even after the use of higher temperature during printing, no drug degradation was observed [Figure 3]. ${ }^{[22]}$ Later, Goyanes et al. further explore the potential of FDM 3DP to produce modified-release 5-aminosalicylic acid (5-ASA, mesalazine) and 4-ASA, loaded tablets. ${ }^{[23]}$ Water-soluble polymer, PVA filaments were loaded with the drugs. An ethanolic solution of 5-ASA and 4-ASA was used to load the commercially available PVA filaments to get the final loading of $0.06 \% \mathrm{w} / \mathrm{w}$ and $0.25 \% \mathrm{w} / \mathrm{w}$, respectively. No chemical interaction between drugs and the filaments was observed, and the drug was found to diffuse passively inside the polymer. $10.5 \mathrm{~mm}$ diameter tablets of both PVA/4-ASA and PVA/5-ASA were printed using an FDM 3D printer [Figure 4]. Goyanes et al. demonstrated the feasibility of using FDM printers to print the drug-loaded tablets at varying concentrations. One important finding of this work is the thermal degradation of the active 4-ASA (50\%) which was confirmed by DSC data. High extrusion temperature, around $210^{\circ} \mathrm{C}$ which is needed to print the tablet from PVA filament, indicated

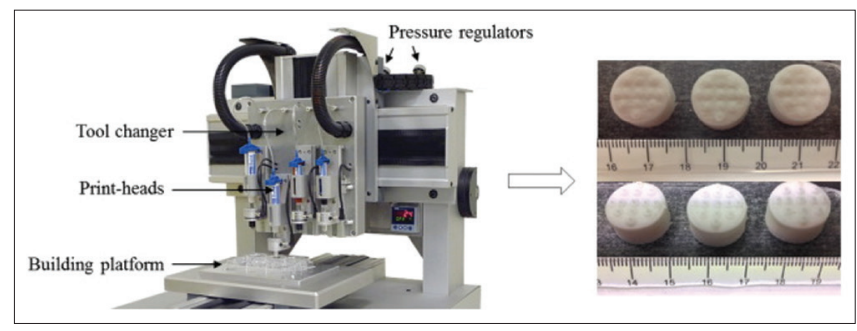

Figure 2: RegenHU three-dimensional printer (left), and image of polypill tablet (right) composed of sustained release compartments (bottom), and immediate release dotted compartment (top). ${ }^{[20]}$ Reprinted with permission 
Charbe, et al.: 3D printed colon drug delivery systems

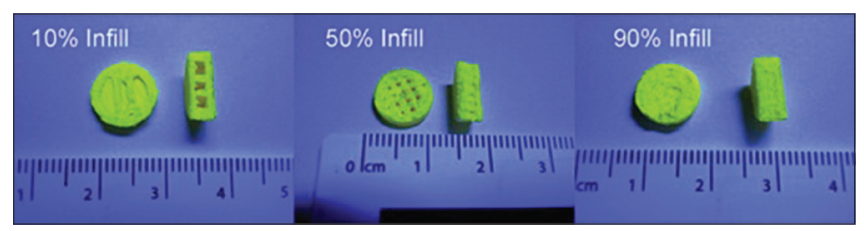

Figure 3: Schematic of infill percentage with fused deposition modeling of tablets. ${ }^{[22]}$ Reprinted with permission

that the method may not be suitable for the drugs when printing at high temperatures exceeding those of the degradation point. However, this problem could be solved using the polymer filament which could enable the printing at temperatures lower than the decomposition temperature of the drug. This approach has the potential to modulate the release of 5-ASA and 4-ASA in the colon using the polymer coats which degrades or dissolves in the colon.

Pietrzak et al. formulated the IR and extended release tablets using FDM 3DP. Pietrzak et al. evaluated the use of methacrylic polymers, Eudragit RL, RS, and E, and cellulosic polymer hydroxypropyl cellulose (HPC) to extrude theophylline-loaded filaments. ${ }^{[24]}$ The major problem as discussed earlier with FDM 3DP is that it is not suitable to the heat liable drugs. To facilitate printing at lower temperatures, up to $7.5 \%$ triethyl citrate, was added as a plasticizer. Considerable lowering of the temperature is not recommended as it could lead to the blockage of the nozzle due to the increased viscosity of the fluid. The drug content of the final printed tablet was found to be $91 \%-95 \%$ of theoretical. As the processing was carried out at a lower temperature, the overall drug degradation is not pronounced as shown by Goyanes et al. for 4-ASA (50\% reduction) using the same printing technique. ${ }^{[23]}$

Despite the advantages and advancement in FDM 3DP, the number of polymers currently available to use in FDM 3DP is limited to PVA and polylactic acid. ${ }^{[25]}$ FDM $3 \mathrm{DP}$ is also of no use for the production of IR tablets, which almost account for $70 \%$ of the tablet formulation. Very recently, Okwuosa et al. developed low-temperature FDM 3DP for the manufacturing of patient-specific IR tablets. ${ }^{[26]}$ In this rare example, Okwuosa et al. used methacrylic polymers to print the tablet. Okwuosa et al. combined the advantages of polyvinylpyrrolidone as an impending polymer with FDM 3DP at low temperatures; this approach holds a potential in expanding the spectrum of drugs that could be used in FDM 3DP. Okwuosa et al. achieved this by bringing 3DP process with hot melt extrusion (HME) in the presence of thermostable filler, talc, which enabled the fabrication of IR tablets at temperatures as low as $110^{\circ} \mathrm{C}$. The integrity of two model drugs (theophylline or dipyridamole) was maintained following HME and FDM 3DP. In this report,

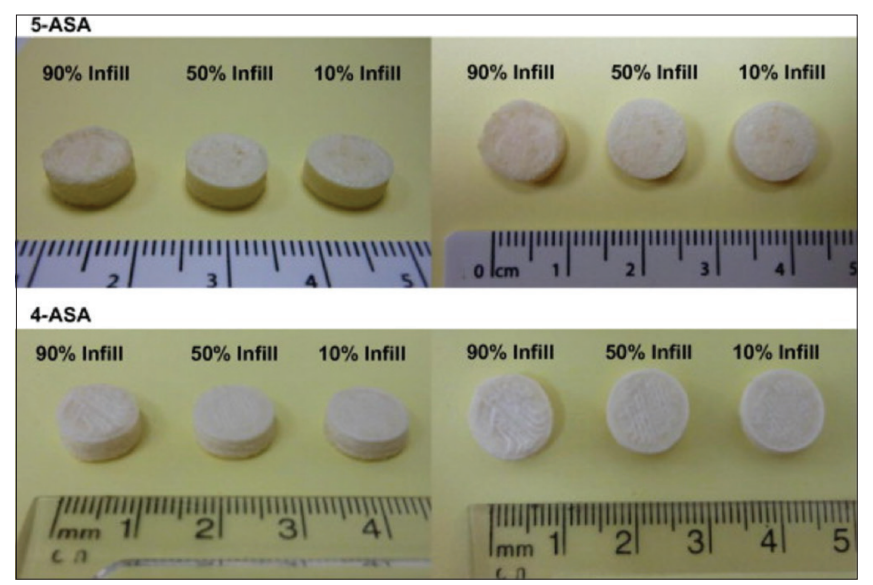

Figure 4: Images of the three-dimensional printed fabricated tablets with different infill percentage. ${ }^{[23]}$ Reprinted with permission

Okwuosa et al. demonstrated the fabrication of IR tablet using a $3 \mathrm{D}$ printer at low temperature, thereby broadening the scope of drugs that could be used with FDM.

Goyanes et al. once again explored the viability of using FDM 3DP technology with HME and fluid bed coating to prepare modified-release budesonide tablet. ${ }^{[27]}$ This was the first time when FDM 3DP combined with HME and fluid bed coating to get the modified released formulation. HME used for the first time to load the budesonide into PVA filaments. The filaments were arranged using the 3D printer into capsule-shaped tablets. Each tablet was made containing $9 \mathrm{mg}$ budesonide at an extrusion temperature of $190^{\circ} \mathrm{C}$. To get the SR, the tablets were then coated with a layer of enteric polymer (Eudragit ${ }^{\circledR}$ L100). The release profile from the tablet was modified using a varying proportion of Eudragit ${ }^{\circledR}$ L100. The final formulation was then compared with the marketed formulations of budesonide through Cortiment ${ }^{\circledR}\left(\right.$ Uceris $\left.^{\circledR}\right)$ and Entocort ${ }^{\circledR}$. It was observed that budesonide was released rapidly from Entocort ${ }^{\circledR}$ in the condition similar to that of upper small intestine, whereas the budesonide release from Cortiment ${ }^{\mathbb{R}}$ was found to be more delayed and slow. However, the newly 3D-printed caplet was found to release the drug in a sustained way along the distal intestine and colon, making it possible to use to prepare the colon release tablets. This work first time demonstrated that HME and film coating could be successfully combined with FDM 3DP to prepare the sustained-release formulation.

FDM-based 3DP was very well exploited by Skowyra et al. to investigate the feasibility of using an FDM-based 3D printer to prepare extended release tablet. The tablet was fabricated using prednisolone-loaded PVA filaments. Various concentrations were achieved in filaments to prepare the tablets of various concentrations. ${ }^{[21]}$ 
Charbe, et al.: 3D printed colon drug delivery systems

Prednisolone was loaded into a PVA filament of $1.75 \mathrm{~mm}$ diameter. Swelling of the PVA filaments was carried out in the methanolic solution of prednisolone. Skowyra et al. demonstrated that PVA filaments have an ability to be fabricated into regular shape tablets using the FDM-based $3 \mathrm{D}$ printer. One important finding of this work suggested that the ethanolic solution of the model drugs was found not suitable for the PVA loading which is contrary to the findings of the work discussed earlier. In vitro, drug release studies have shown that the around $80 \%$ of the drug release occurred after $12 \mathrm{~h}$ for 2 and $3 \mathrm{mg}$ tablet and $18 \mathrm{~h}$ for $4,5,7.5$, and $10 \mathrm{mg}$. It was observed that around $100 \%$ drug release took place after $18 \mathrm{~h}$ during in vitro studies. Skowyra et al. were able to extend the in vitro drug release from 3D-printed tablets by up to $24 \mathrm{~h}$, suggesting its application for the development of the colon targeted drug delivery system.

Khaled et al. demonstrated that the complex medication regimes can be combined in a single tablet to form the polypill using 3D extrusion-based printing technique. ${ }^{[28]}$ Roberts et al. have developed multi-active tablets with well-defined and separate drug release profiles for three different drugs; captopril, nifedipine, and glipizide [Figure 5]. Roberts et al. were able to incorporate two different concepts of drug release through osmotic release for captopril and SR for nifedipine and glipizide in the single tablet. One of the most important highlights of this work is the use of common excipients at normal temperature, thereby avoiding the drug and excipient degradation at a higher temperature. Roberts et al. also confirmed that the captopril release from the tablet followed zero-order release and nifedipine and glipizide followed first-order release. Captopril release was found to be affected by the hydroxypropyl methylcellulose (HPMC) concentration; higher HPMC concentration caused delayed drug release. A similar effect of HPMC was observed on

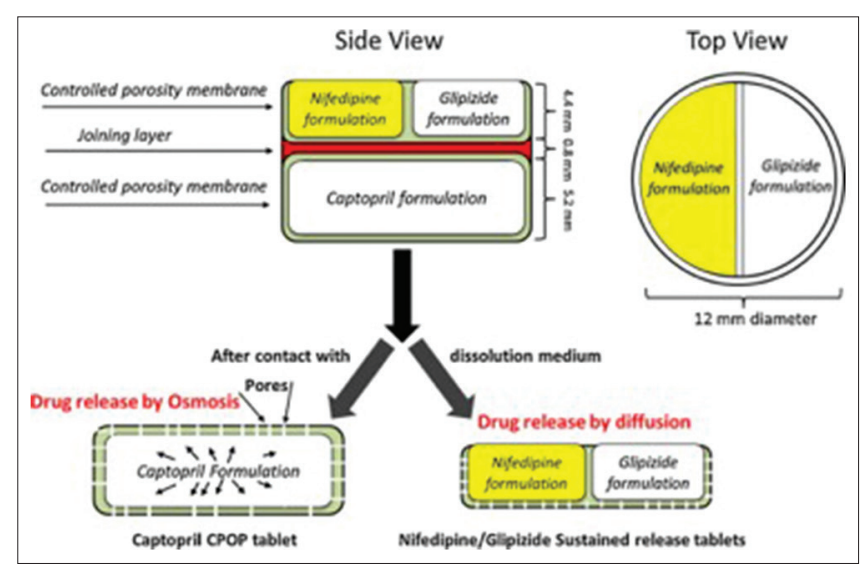

Figure 5: Structural diagram of the three-dimensional printed tablets having an osmotic release for captopril and sustained release for nifedipine and glipizide. ${ }^{[28]}$ Reprinted with permission nifedipine and glipizide released from the sustained released compartment. This tablet formulation will be very useful for the patient suffering from hypertension and diabetes.

In 1999, Rowe et al. emphasized the ability of 3D fabrication to produce four different complex dosage forms by producing tablets with immediate and extended, enteric dual pulsatory, and breakaway drug-release profiles. ${ }^{[29]}$ Rowe et al. demonstrated the flexibility of the $3 \mathrm{D}$ printers to produce the tablets with different release profiles which are not possible by classical compression technique. Yu et al. for the first time printed doughnut-shaped multilayered drug delivery devices with variations in the drug and materials affecting the drug release. Liner release from the dosage form was achieved using sustained released polymers at varying gradient concentrations. Yu et al.'s doughnut-shaped drug delivery devices are shown in Figure 6. ${ }^{[30]}$ Doughnut shape was chosen because mathematical model proposed that tablet with a central hole(s) would provide a constant linear drug release surface area. ${ }^{[31]}$ This shape had been previously shown to produce zero-order release by controlling surface area during erosion; ${ }^{[32]}$ however, manufacturing these tablet geometries required difficult compression processes. Acetaminophen was used as the model drug, and HPMC and ethylcellulose were used as the released retardant material. Yu et al. made a structure with upper and lower layers made up of ethylcellulose to get impermeable layers. The inner core was made by mixing drug with [shown in grey in Figure 6] $2 \%$ ethyl cellulose to create a slower release rate from the exposed surface. Theoretically, doughnut shape geometry allows a decrease in the surface area due to the outward releasing portion and the increase in the surface area of the inward releasing portion to produce a zero-order release. It was confirmed that the zero-order release was seen for the printed tablet with the drug release rate varying with the thickness of the impermeable membrane and tablet height. It was also confirmed that no burst release was seen with doughnut-shaped tablet which is the main issue with many SR tablets.

Khaled et al. utilized 3D extrusion printing to print a novel polypill tablet capable of satisfying regulatory tests

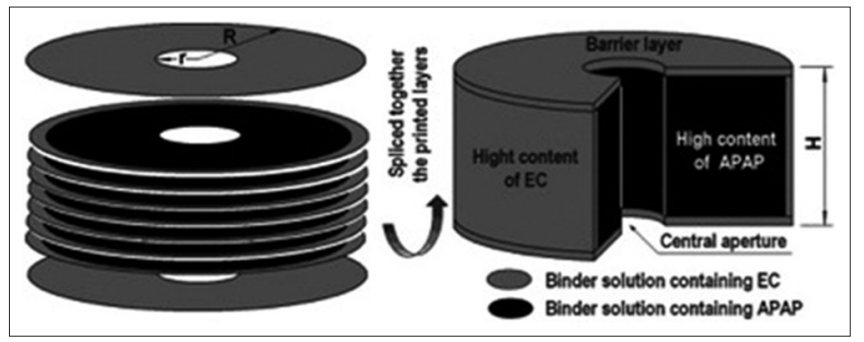

Figure 6: Doughnut-shaped tablet for zero-order release. ${ }^{[30]}$ Reprinted with permission 
and matching the release of the commercially available tablet (guaifenesin bi-layer tablet). ${ }^{[33]}$ For the first time, Khaled et al. used the excipients similar to that used in the commercial formulation. HPMC 2208 (Methoce $^{\mathrm{TM}}$ K100M Premium) and poly(acrylic acid) (PAA) (Carbopol ${ }^{\circledR}$ 974P NF) were used as a hydrophilic matrix for an SR layer. Hypromellose $^{\circledR}$ (HPMC 2910) was used as a binder while microcrystalline cellulose (MCC) (Pharmacel ${ }^{\circledR}$ 102) and sodium starch glycolate (SSG) $\left(\right.$ Primojel $^{\circledR}$ ) were used as disintegrants for an IR layer. To get the IR layer, guaifenesin powder was mixed with SSG and MCC, whereas to get the sustained layer, guaifenesin powder was mixed with HPMC 2208. HPMC 2910 (1\%, w/v) was blended with the powdered drug to obtained the homogenous paste. Similar to Khaled et al., the important highlight of this work is the use of the room temperature extrusion for 3DP. ${ }^{[28]}$ The room temperature extrusion was not only suitable for the distinct excipient used for 3DP of polypill but also allowed the use of the drugs liable to the heat degradation. The printed formulations were found to have the comparable physical and mechanical parameters when compared with the marketed formulation and were within the acceptable range as defined by the United States Pharmacopoeia.

This literature survey of 3D-printed tablets essentially proves the emerging success of the 3D-printed tablets. In future, these formulations could be easily remolded to develop the colon specific tablets by varying not only the polymers but also the thickness of the polymers to achieve personalized colon targeted drug delivery systems.

\section{COLON-SPECIFIC DRUG DELIVERY USING THREE-DIMENSIONAL-PRINTED TABLETS}

The various strategies for CDDS include covalent linkage of a drug with a carrier (e.g., azo bond conjugates, glycoside conjugates, glucuronide conjugates, cyclodextrins conjugates, dextran conjugates, amino acid conjugates), coating with $\mathrm{pH}$-sensitive and biodegradable polymers, formulation of timed released systems, embedding inside the matrix, embedding inside the $\mathrm{pH}$ sensitive matrix, exploitation of carriers that are degraded specifically by colonic bacteria, times released system, redox-sensitive polymers, bioadhesive systems, and osmotic controlled drug delivery systems.

To release budesonide in the upper small intestine and continues till colon, Goyanes et al. utilized FDM 3DP technology with HME and fluid bed coating. ${ }^{[27]}$ Goyanes et al. successfully loaded budesonide into commercially available PVA filaments. Drug released properties of
3D-printed budesonide tablets were compared with the marketed preparation like Entocort. It was observed that 3D-printed budesonide tablet was found to continue drug release in a sustained manner till it reaches the colon. The drug release from this 3D-printed budesonide tablet starts from the mid-small intestine. This work has successfully established the method and usefulness of FDM 3DP, HME, and fluid bed coating of the tablet made from the budesonide-loaded PVA filaments. This method could be successfully adapted to prepare the CDDS by coating the drug-loaded filament tablet with a polymer like Eudragit L100. Similarly, Khaled et al., who utilized HPMC 2208 (Methocel $^{\mathrm{TM}}$ K100M Premium) and PAA $\left(\right.$ Carbopol $^{\circledR}$ 974P NF) as a hydrophilic matrix for an SR layer, is worth to try with EUDRAGIT ${ }^{\circledR}$ Polymer L 30 D-55 (dissolution above $\mathrm{pH}$ 5.5), EUDRAGIT ${ }^{\circledR}$ Polymer L 100-55 (dissolution above $\mathrm{pH}$ 5.5), EUDRAGIT ${ }^{\circledR}$ Polymer L 100 (dissolution above $\mathrm{pH}$ 6.0), and EUDRAGIT ${ }^{\circledR}$ Polymer L 12.5 (dissolution above $\mathrm{pH}$ 6.0). ${ }^{[33]}$ Use of EUDRAGIT $^{\circledR}$ polymers coating permits drug release in the colon.

Targeted drug release is not only required for the local treatment of gut diseases such as Crohn's disease, ulcerative colitis, or intestinal cancer but sometimes is also required to protect the drugs from the acidic condition of the upper GIT. The gastro-resistance coating protects the dosage form from degradation. To release the drug in the colon, the most favored synthetic polymers is Eudragit ${ }^{\circledR}$ FS30 D. Eudragit ${ }^{\circledR}$ FS30 D offers the technical advantage of high flexibility in coating; however, other polymers such as Eudragit ${ }^{\mathbb{R}}$ L100 and Eudragit ${ }^{\mathbb{R}}$ S100 are also used before in the development of CDDS. ${ }^{[3]}$ Similarly, natural polymers such as pectin, chitosan, chondroitin sulfate, dextran, cyclodextrin, inulin, and xanthan could be utilized in varying thickness to develop the CDDS. Taking clue from the Rowe et al. ${ }^{[29]} 3 \mathrm{D}$ fabrication technique could be used to produce complex colon-specific formulations by producing tablets with immediate and extended, enteric dual pulsatory, dual pulsatory, and breakaway release profiles.

Yu et al. printed tablets in a doughnut shape; this shape had been previously shown to produce zero-order release by controlling surface area during erosion. ${ }^{[30]} \mathrm{Yu}$ et al. successfully use this shape to produce the linear drug release. Top and bottom layers of this doughnut-shaped tablet were made from impermeable ethylcellulose polymer. These top and bottom layers of varying thickness could facilitate the drug release in the colon. The inner core could be prepared using an active drug specific for colon absorption and treatment with the binder suitable for the slower release in the colon [Figure 6]. 
Goyanes et al. first evaluated the use of FDM with the incorporation of fluorescein into commercially available PVA filaments. To take care of the individual patient needs, Goyanes et al. demonstrated that the different release profile of drugs is achievable using this technology. The most important highlight of this work is the fabrication of the tablets with different amount of infills with different release profile. Successful development of the different release profile and infill's evident the possibility of the fabrication of colon targeted formulations. ${ }^{[2]}$ Goyanes et al. prepared the 3D-printed hollow shell. These hollow shells could be used to fill the drugs for colonic release. ${ }^{[2]}$ The method developed by Khaled et al. to combine separate release profiles such as osmotic release and SR in a single polypill could be modulated further to develop the colon-specific tablets. ${ }^{[28]}$ Tablet formulation with difference release profile is very useful for the patients who need to take the two different drugs, to be released at the two different sites of GIT, for example, drug release in stomach and colon or drug release in the intestine and colon. Similarly, in another approach of Roberts et al., in which they developed five-in-one dose combination, polypill with defined IR and SR profiles offers a valuable clue for the development of the colon release formulations. This information is especially valuable in case if we need to coformulate the drug for colon release with the one which needs to be released immediately in the stomach or in the intestine. ${ }^{[20]}$

Due to the advancement in the personalized medicine during the last decade, there is an increased demand for the reliable production of personalized formulations which could be prepared by the bedside. Personalization of the formulation is essential because one dose does not suit all; this is also applicable to the patients who need to treat their colon. To take care of this issue, Pietrzak et al. evaluated the use of methacrylic polymers, Eudragit RL, RS, and E, and HPC to extrude theophylline-loaded filaments. ${ }^{[2]}$ This approach is very useful to prepare the $3 \mathrm{D}$-printed colon release tablets in case if we could manage to use the colon targeted drugs, gene products, and polymer Eudragit RL and RS with FDM printers at high temperature. Polymer EUDRAGIT $^{\circledR}$ RL and RS with cationic groups enable time-controlled release of the drug by $\mathrm{pH}$-independent swelling, and hence, they are very useful for delayed and sustained drug release formulations. On the other hand, Okwuosa et al. developed the lower temperature FDM 3DP to create patient-specific IR tablets. This approach combines the advantages of PVA as an impending polymer with FDM 3DP at low temperatures. This method will be very useful for the drugs which are heat sensitive or unstable at a higher temperature.
Fast and reliable personalization of the dosage formulation is the biggest challenge in the development of the personalized therapy. Skowyra et al. investigated the FDM-based 3D printer to fabricate extended release tablet using prednisolone-loaded PVA filaments. Finding of this work would be useful to develop the targeted colon-specific delivery system by loading the PVA filament with prednisolone or other colon-specific drugs to create the tablet and simultaneously coating the same with chitosan or cellulose acetate phthalate which dissolves at the $\mathrm{pH}$ 6.0. ${ }^{[21]}$ Same approach could be used to develop colon targeting system based on enteric-coated matrix tablets which could exploit $\mathrm{pH} 0$ and time-dependent release function. $\mathrm{pH}$ - and time-dependent release are possible using the polymers such as hydroxyethylcellulose, hydrophilic swellable polymer, ethyl cellulose, or microcrystalline cellulose polymers, in which drugs such as theophylline could be dispersed. Eudragit S100, a methacrylic acid copolymer soluble at $\mathrm{pH} 7$, can be used as the $\mathrm{pH}$-sensitive coating polymer. ${ }^{[35]}$

The capability of guar gum coating to develop CDDS is already evaluated and could be the good choice for 3DP. For example, in the treatment of colon inflammation and pain, flurbiprofen is the preferred nonsteroidal anti-inflammatory drug. However, unwanted excess accumulation of flurbiprofen in the stomach and small intestine leads to the side effects such as ulcers and internal bleeding. ${ }^{\left[{ }^{[6]}\right.}$ To reduce the nonspecific side effects, the most appropriate approach is the release of flurbiprofen in colon. Guar gum, which is a naturally occurring galactomannan polysaccharide obtained from the endosperm of the guar plant Cyamopsis tetragonoloba, could be used to coat the core of flurbiprofen for its release in colon. ${ }^{[3]}$ Similarly, 3D printers could be used to coat the 5-florouracil core for colonic release using natural polysaccharides such as gaur gum, dextran, and $\mathrm{pH}$-sensitive polymers such as Eudragit L100 and Eudragit S100. ${ }^{[38]}$

CDDS is not only useful for the local absorption but also a valuable tool for systemic absorption of the drugs, for example, colonic absorption of anti-inflammatory drugs in rheumatoid arthritis. The justification for the development of a polysaccharide-based CDDS is the various polysaccharide-specific bacterial enzymes present in the human colon, for example, amylase, pectinase. Polysaccharides in CDDS are used in the matrix, on the periphery coat or linked with the drugs to be cleaved by the enzymes. For example, dietary source for the chondroitin sulfate source is the meat and is the substrate for the Bacteroides thetaiotaomicron and Bacteroides ovatus. ${ }^{[3]}$ Chondroitin sulfate was exploited by Ramasamy et al. for the colonic drug delivery of aceclofenac. ${ }^{[40]}$ Ramasamy et al. 
used HPMC K-100 as a hydrophilic polymer, and tablet was manufactured by wet granulation technique using starch mucilage as a binding agent. $\mathrm{pH}$-dependent polymers such as Eudragit L100 and Eudragit S100 were used to protect the core from the acidic condition. Drug release was found to increase when chondroitin sulfate concentration in the colon increases and caused polymer degradation. 3DP technique in conjugation with chondroitin sulfate and Eudragit L100 and Eudragit S100 combination could be used to fabricate the colon-specific release of aceclofenac. Similarly, aceclofenac could be targeted to the colon using natural polymer-like chitosan. Chitosan is not only completely degradable by colonic bacteria into harmless metabolites, but also it is relatively inexpensive and inert making it useful excipient for dose formulations. For the treatment of irritable bowel syndrome, Eudragit S100 and chitosan could be combined in 3DP of colon-specific ondansetron tablets. In this combination, Eudragit S100 get solubilized depending on the $\mathrm{pH}$, whereas the chitosan undergoes microbial degradation to release the drug. ${ }^{[4]}$ The same technique could be used to prepare the CDDS for indomethacin and curcumin. ${ }^{[42]}$ Tramadol hydrochloride which is a centrally acting opioid analgesic agent could be released in the colon by forming the matrix core with hydrophilic material and external coating of $\mathrm{pH}$-dependent polymer Eudragit S100. ${ }^{[43]}$

The release of the water-soluble drugs from water-soluble polymers leads to the rapid onset of the action. Use of hydrophobic $\mathrm{pH}$-independent polymers such as Eudragit RSPO and RLPO instead of hydrophilic polymers would be the appropriate choice for highly water-soluble drugs. These polymers are the most logical choice if such water-soluble drugs are needed to be released in the colon, for example, naproxen could be mix with Eudragit RSPO and RLPO to get the matrix core. This matrix core further coated with $\mathrm{pH}$-dependent polymer like Eudragit S100 could release drugs in colon. ${ }^{[44]}$ Matrix preparation with Eudragit RSPO and RLPO however may not useful for the water-insoluble drugs.

Ketorolac tromethamine could also be delivered to the colon by preparing 3D-printed tablets with double coats. $3 \mathrm{D}$ printer could be used to deposit the inner coat of time-controlled HPMC K100M polymer and the outer coat of $\mathrm{pH}$-sensitive Eudragit S100 polymer. Outer coat will be useful to release the drug in the colon and inner coat will be useful for sustained drug release. ${ }^{[45]}$ Similarly, colon targeted delivery of metronidazole is also possible using 3DP technology. Metronidazole tablet could be prepared using it along with suitable swelling agents to provide $\mathrm{pH}$-sensitive pulsatile drug delivery. Eudragit ${ }^{\circledR}$ S100 (ES) and ethyl cellulose could be used as the coating polymers to prevent initial drug release in the gastric region. In case, if metronidazole has to be combined with other agents such as clindamycin or ornidazole which needs to be released in stomach and intestine, then Khaled et al. or Khaled et al. approach would be useful to prepare the polypills with different release profile. ${ }^{[28,33]}$

Sangalli et al. have developed a very novel drug delivery system termed as Chronotopic ${ }^{\mathrm{TM}}$, specifically for the delayed drug release in the colon. ${ }^{[4]}$ The drug core is coated with a swellable hydrophilic polymer responsible for the lag phase before the drug release actually starts. As soon as the hydrophilic polymer comes in contact with the aqueous fluid, it swells and transits into the glassy-rubbery material. In this state, it becomes more permeable and starts to erode. This causes the delayed drug release over the period of time depending on the thickness of the polymer applied. By applying a gastro-resistant film on the above-described hydrophilic polymer-coated, the variability in gastric emptying time can be overcome and a colon-specific release could be attained as per the time-dependent approach. This could be the very useful approach in the 3DP of tablets containing drugs for colon release.

Again, considering GIT environment and transit time, rational colonic drug delivery system could be developed using $3 \mathrm{D}$ printer, for example, swelling matrix core containing pectin, HPMC, MCC, and the active drug could be prepared. ${ }^{[4]}$ The swellable core can then be subjected to an inner $\mathrm{pH}$-sensitive and an outer semipermeable membrane coat. Pectin in this combination is a nontoxic soluble carbohydrate, degradable by colonic bacterial enzymes called pectinolytic. In addition, HPMC is a release modulator required to develop a controlled release system. This dual-coated formulation releases its contents at $\mathrm{pH} 1.5$ or 5.5 for $12 \mathrm{~h}$. However, it provides reproducible and controlled drug release in $\mathrm{pH} 6.8$, with an initial lag time of about $6 \mathrm{~h}$.

Time- and pH-dependent release of tinidazole in the colon are achievable using the technique described by Naikwade et al. ${ }^{[48]}$ Tinidazole core can be prepared using swellable and $\mathrm{pH}$-dependent polymers such as HPMC (HPMC K4M and K15M) and Eudragit coating (Eudragit L100 and S100), respectively. Swellable polymers when comes in contact with aqueous medium starts to swell forming a rubbery coat which then starts to erode and release the drug, whereas the $\mathrm{pH}$-dependent core offers the initial protection from the gastric acidic juice. Prepared tablets need to be enteric coated to overcome variability in gastric emptying time and delay in the release. Enteric coating is also essential to 
avoid the unwanted side effects at nonspecific sites such as stomach and small intestine and is very useful to increase the sustained localized action in the colon. Polymer like acrylic resin which is soluble at $\mathrm{pH}>5$ along with HPMC as film-coating agent could be used using a $3 \mathrm{D}$ printer to prepare the colon-specific tablet.

Overall, various natural polymers have been explored for colon drug release formulation, for example, pectin, guar gum, amylase, inulin, and dextran, chitosan, and chondroitin sulfate. The $\mathrm{pH}$ gradient across the GIT leads to the development of the various synthetic polymers which are soluble only at the higher colonic $\mathrm{pH}$, for example, copolymers of methacrylic acid, methyl methacrylate, and ethyl acrylates. The focus of today's pharmacotherapy is based on the patient compliance and most of the sustained, extended, or targeted formulation makes use of these polymers. The main use of the polysaccharide in drug delivery systems is the time-dependent drug release and protection of the bioactive from the hostile conditions of the upper GIT. Interest in the natural biodegradable polymers has grown significantly because they are safe, nontoxic, economical, naturally occurring, abundantly available with varying chemical compositions, and most importantly, they are chemically inert. Because of ease of approval and nontoxic nature, natural polysaccharides could be the polymer of choice in the 3DP of colon-specific tablets.

\section{CONCLUSION}

The modern pharmaceutical industry is not only involved in the manufacturing and marketing of the drugs, but they are constantly involved in the research and development. The constant pursuit for the new products has led to the development of the unique excipients and active agents. Innovations in pharmaceutical engineering have considerably improved manufacturing speed and quality of the end-product. Although these advancements have very well served the pharmaceutical industry and patients' needs, it has limited application in terms of creating dosage forms with different or targeted release profiles. Its application in producing the personalized/tailored dosage forms is also limited.

3DP of oral tablet dosage formulation seems to be the most exciting application of 3DP in pharmaceutical industry. Conventionally, compressed tablets are made from a compression of the mixture of the drug and the excipients. However, this approach is not very suitable for complex release profile and for the drugs and excipients which could not stand the high pressure. 3D printer could solve this issue by facilitating the print of polymer over the powder bed in layers of $200 \mu \mathrm{m}$ thickness or less, thereby creating a barrier between the active drugs to allow the controlled release. ${ }^{[49]} 3 \mathrm{D}$ printers also allow the fabrication of complex geometries such as doughnut shape, oval shape, or hollow tablets topped with barrier layers that could be modulated to get zero-order or linear release of the drug. $3 \mathrm{D}$ printers have the potential to fabricate a single pill with multiple drugs in it.

The ability to manufacture a single tablet with multiple drugs and different release profiles (polypill) not only reduces the number of tablets to be taken at a time but also increases the adherence to the dose regimen. Polypills are certainly the most valued formulation in case if drugs need to have the independent release profiles. Khaled et al. demonstrated the fabrication of a five-drug polypill having different release profile using 3DP technique. ${ }^{[20]} \mathrm{A}$ $3 \mathrm{D}$ printer has the unique ability to manufacture complex geometries with multiple materials to form the solid objects. In this example, five-drug polypill exploits only one particular version of $3 \mathrm{DP}$, but it is worth to remember here that there are several currently recognized distinct methods of 3DP available.

Drug release from the CDDS is the interplay between the swelling and the polymer agents which in turn depends on the colon environment, for example, $\mathrm{pH}$ and enzymes. The thickness of polymer coat is also an important limiting factor in colon release. Polymer coat mostly protects the core to disintegrate and release the drugs in higher $\mathrm{pH}$ of stomach and intestine. This polymer dissolves and disintegrates at colonic $\mathrm{pH}$, thereby releasing the drugs for absorption. The thickness of the polymers coat could be precisely managed by depositing it using 3D printers. Enzyme-, pH-, and time-dependent release of the drug in the colon are possible using the various polymers (having a different solubility at different $\mathrm{pH}$ ) in varying thickness.

The recent approval of the first 3D-printed tables has not only hinted the future role of $3 \mathrm{D}$ printers in tablet manufacturing but also ascertained that it has the capacity to meet the stringent FDA guidelines. 3D-printed formulations are definitely a potential futuristic innovation; however, to be the replacement for the traditional bulk manufacturing units, not only the improvement in the technique is needed but also it has to overcome the issues related to the approvals. Application of 3D-printed tablets (polypills) in personalized or tailored therapy is possible only if it will be able to avoid the regulatory issues. The current FDA guidelines consider each 3D printer as a separate manufacturing machine and each pharmacy as 
the manufacturing unit. This makes it necessary to get the necessary approvals for the printer and for the pharmacy. Under the current situation, approving all the pharmacies and other printing facilities is a difficult task. To make this as a realistic approach, radical changes are required not only in the 3DP technique but also needs to redraft the regulatory guidelines to accommodate 3D printers and printing sites.

Ultimately, the cost to clinical benefit ratio will guide the $3 \mathrm{D}$ printers toward the mainstream use. The cost of manufacturing the 3D-printed tables should not be high enough to make it unaffordable for the patients, for example, manufacturing cost of the Spritam; a fast-dissolving 3D-printed tablet is higher than the currently available methods. If drug release, bioavailability, and efficacy remain the same, then the justification of the increased cost is essential. Similarly, if the tablet manufactured by the 3DP technology and by the conventional methods serves the same function, then the advantage of the 3D-printed tablets should outweigh the advantage of tablets manufactured by the traditional method. For example, 3D-printed polypills are the motivational innovation in drug delivery, but we should not forget the fact that any new combination needs to prove its safety, efficacy, and equivalency through controlled clinical studies. In future, local pharmacies with 3DP facilities will be common. Manufacturing of the dosage formulations by the pharmaceutical giants will be soon replaced by the on-demand printing of the formulation. These changes will not only increase the patient compliance but would also be more cost-effective in terms of bespoke manufacturing and distribution.

We envision that 3D-printed tablets developed using approaches described in this review could soon be the common practice for the fabrication of personalized colon targeted drug delivery systems.

\section{Financial support and sponsorship}

Nil.

\section{Conflicts of interest}

There are no conflicts of interest.

\section{REFERENCES}

1. Eisenstein M. First 3D-printed pill. Nat Biotechnol 2015;33:1014.

2. Ventola CL. Medical applications for 3D printing: Current and projected uses. P T 2014;39:704-11.

3. Krishna R, Garg A, Jin B, Keshavarz SS, Bieberdorf FA, Chodakewitz J, et al. Assessment of a pharmacokinetic and pharmacodynamic interaction between simvastatin and anacetrapib, a potent cholesteryl ester transfer protein (CETP) inhibitor, in healthy subjects. Br J Clin Pharmacol 2009;67:520-6.
4. Sandle GI. Salt and water absorption in the human colon: A modern appraisal. Gut 1998;43:294-9.

5. Chourasia MK, Jain SK. Pharmaceutical approaches to colon targeted drug delivery systems. J Pharm Pharm Sci 2003;6:33-66.

6. Tromm A, Möllmann H, Barth J, Hochhaus G, Krieg M, Bigalke C, et al. Pharmacokinetics and rectal bioavailability of hydrocortisone acetate after single and multiple administration in healthy subjects and patients. J Clin Pharmacol 2001;41:536-41.

7. Philip AK, Philip B. Colon targeted drug delivery systems: A review on primary and novel approaches. Oman Med J 2010;25:79-87.

8. McLeod AD, Friend DR, Tozer TN. Glucocorticoid-dextran conjugates as potential prodrugs for colon-specific delivery: Hydrolysis in rat gastrointestinal tract contents. J Pharm Sci 1994;83:1284-8.

9. Criscuoli V, Modesto I, Orlando A, Cottone M. Mesalazine for the treatment of inflammatory bowel disease. Expert Opin Pharmacother 2013;14:1669-78.

10. Carrette O, Favier C, Mizon C, Neut C, Cortot A, Colombel JF, et al. Bacterial enzymes used for colon-specific drug delivery are decreased in active Crohn's disease. Dig Dis Sci 1995;40:2641-6.

11. Rubinstein A. Approaches and opportunities in colon-specific drug delivery. Crit Rev Ther Drug Carrier Syst 1995;12:101-49.

12. Vassallo M, Camilleri M, Phillips SF, Brown ML, Chapman NJ, Thomforde GM. Transit through the proximal colon influences stool weight in the irritable bowel syndrome. Gastroenterology 1992;102:102-8.

13. Wostmann BS. The germfree animal in nutritional studies. Annu Rev Nutr 1981;1:257-79.

14. Sinha VR, Kumria R. Microbially triggered drug delivery to the colon. Eur J Pharm Sci 2003;18:3-18.

15. Scheline RR. Metabolism of foreign compounds by gastrointestinal microorganisms. Pharmacol Rev 1973;25:451-523.

16. Hergenrother RW, Wabers HD, Cooper SL. The effect of chain extenders and stabilizers on the in vivo stability of polyurethanes. J Appl Biomater 1992;3:17-22.

17. kumar JR, Muralidharan S, Dhanaraj SA, Umadevi SK. A novel drug delivery systems of colon targeted: A review. J Pharm Sci Res 2013;5:42-7.

18. Singh R, Sahay A, Muzzio F, Ierapetritou M, Ramachandran R. A systematic framework for onsite design and implementation of a control system in a continuous tablet manufacturing process. Comput Chem Eng 2014;66:186-200.

19. Sandler N, Määttänen A, Ihalainen P, Kronberg L, Meierjohann A, Viitala $\mathrm{T}$, et al. Inkjet printing of drug substances and use of porous substrates-towards individualized dosing. J Pharm Sci 2011;100:3386-95.

20. Khaled SA, Burley JC, Alexander MR, Yang J, Roberts CJ. 3D printing of five-in-one dose combination polypill with defined immediate and sustained release profiles. J Control Release 2015;217:308-14.

21. Skowyra J, Pietrzak K, Alhnan MA. Fabrication of extended-release patient-tailored prednisolone tablets via fused deposition modelling (FDM) 3D printing. Eur J Pharm Sci 2015;68:11-7.

22. Goyanes A, Buanz AB, Basit AW, Gaisford S. Fused-filament 3D printing (3DP) for fabrication of tablets. Int J Pharm 2014;476:88-92.

23. Goyanes A, Buanz AB, Hatton GB, Gaisford S, Basit AW. 3D printing of modified-release aminosalicylate (4-ASA and 5-ASA) tablets. Eur J Pharm Biopharm 2015;89:157-62.

24. Pietrzak K, Isreb A, Alhnan MA. A flexible-dose dispenser for immediate and extended release 3D printed tablets. Eur J Pharm Biopharm 2015;96:380-7.

25. Goyanes A, Robles Martinez P, Buanz A, Basit AW, Gaisford S. Effect of geometry on drug release from 3D printed tablets. Int J Pharm 2015;494:657-63.

26. Okwuosa TC, Stefaniak D, Arafat B, Isreb A, Wan KW, Alhnan MA. A lower temperature FDM 3D printing for the manufacture of patient-specific immediate release tablets. Pharm Res 2016;33:2704-12.

27. Goyanes A, Chang H, Sedough D, Hatton GB, Wang J, Buanz A, et al. Fabrication of controlled-release budesonide tablets via desktop (FDM) 3D printing. Int J Pharm 2015;496:414-20. 
28. Khaled SA, Burley JC, Alexander MR, Yang J, Roberts CJ. 3D printing of tablets containing multiple drugs with defined release profiles. Int J Pharm 2015;494:643-50.

29. Rowe CW, Katstra WE, Palazzolo RD, Giritlioglu B, Teung P, Cima MJ. Multimechanism oral dosage forms fabricated by three dimensional printing. J Control Release 2000;66:11-7.

30. Yu DG, Branford-White C, Ma ZH, Zhu LM, Li XY, Yang XL. Novel drug delivery devices for providing linear release profiles fabricated by 3DP. Int J Pharm 2009;370:160-6.

31. Cleave JP. Some geometrical considerations concerning the design of tablets. J Pharm Pharmacol 1965;17:698-702.

32. Sundy E, Danckwerts MP. A novel compression-coated doughnut-shaped tablet design for zero-order sustained release. Eur J Pharm Sci 2004;22:477-85.

33. Khaled SA, Burley JC, Alexander MR, Roberts CJ. Desktop 3D printing of controlled release pharmaceutical bilayer tablets. Int J Pharm 2014:461:105-11.

34. Nikam VK, Kotade KB, Gaware VM, Dolas RT, Dhamak KB, Somwanshi SB, et al. Eudragit a versatile polymer: A review. Pharmacol Newsl 2011;1:152-64.

35. Alvarez-Fuentes J, Fernández-Arévalo M, González-Rodríguez ML, Cirri M, Mura P. Development of enteric-coated timed-release matrix tablets for colon targeting. J Drug Target 2004;12:607-12.

36. Philip AK, Dubey RK, Pathak K. Optimizing delivery of flurbiprofen to the colon using a targeted prodrug approach. J Pharm Pharmacol 2008;60:607-13.

37. Vemula SK, Bontha VK. Colon targeted guar gum compression coated tablets of flurbiprofen: Formulation, development, and pharmacokinetics. Biomed Res Int 2013;2013:287919.

38. Rai G, Yadav AK, Jain NK, Agrawal GP. Enteric-coated epichlorohydrin crosslinked dextran microspheres for site-specific delivery to colon. Drug Dev Ind Pharm 2015;41:2018-28.

39. Salyers AA. Energy sources of major intestinal fermentative anaerobes. Am J Clin Nutr 1979;32:158-63.
40. Ramasamy T, Subbaih Khandasamy U, Shanmugam S, Ruttala H Formulation and evaluation of chondroitin sulphate tablets of aceclofenac for colon targeted drug delivery. Iran J Pharm Res 2012;11:465-79.

41. Jose S, Dhanya K, Cinu TA, Aleykutty NA. Multiparticulate system for colon targeted delivery of ondansetron. Indian J Pharm Sci 2010;72:58-64.

42. Elkhodairy KA, Elsaghir HA, Al-Subayiel AM. Formulation of indomethacin colon targeted delivery systems using polysaccharides as carriers by applying liquisolid technique. Biomed Res Int 2014;2014:704362.

43. Kadiyam R, Muzib YI. Colon specific drug delivery of tramadol $\mathrm{HCl}$ for chronotherapeutics of arthritis. Int J Pharm Investig 2015;5:43-9.

44. Mehta R, Chawla A, Sharma P, Pawar P. Formulation and in vitro evaluation of Eudragit S-100 coated naproxen matrix tablets for colon-targeted drug delivery system. J Adv Pharm Technol Res 2013;4:31-41.

45. Vemula SK. Formulation and pharmacokinetics of colon-specific double-compression coated mini-tablets: Chronopharmaceutical delivery of ketorolac tromethamine. Int J Pharm 2015;491:35-41.

46. Sangalli ME, Maroni A, Zema L, Busetti C, Giordano F, Gazzaniga A. Invitro and in vivo evaluation of an oral system for time and/or site-specific drug delivery. J Control Release 2001;73:103-10.

47. Talukder RM, Fassihi R. Development and in-vitro evaluation of a colon-specific controlled release drug delivery system. J Pharm Pharmacol 2008;60:1297-303.

48. Naikwade SR, Kulkarni PP, Jathar SR, Bajaj AN. Development of time and $\mathrm{pH}$ dependent controlled release colon specific delivery of tinidazole. Daru J Pharm Sci 2008;16:119-27.

49. Gross BC, Erkal JL, Lockwood SY, Chen C, Spence DM. Evaluation of $3 \mathrm{D}$ printing and its potential impact on biotechnology and the chemical sciences. Anal Chem 2014;86:3240-53. 